S. Villa, R. M. Ceriani, B. E. L. Cerabolini \& S. Pierce

\title{
Germination response across populations of the stenoendemic chasmo- phyte Campanula raineri (Campanulaceae)
}

\begin{abstract}
Villa, S., Ceriani, R. M., Cerabolini, B. E. L. \& Pierce S.: Germination response across populations of the stenoendemic chasmophyte Campanula raineri (Campanulaceae) [In Magrini, S. \& Salmeri, C. (eds), Mediterranean plant germination reports - 3]. Fl. Medit. 31: 267-270. 2021. http://dx.doi.org/10.7320/FlMedit31.267

This study describes a successful germination protocol for Campanula raineri (Campanulaceae), a protected rock-face dwelling species, endemic to the Italian calcareous Prealps. Germination tests were performed on seed lots from nine different populations across the geographical range of the species. Mean germination rates higher than $80 \%$ were reached within 28 days for almost all of the populations under analysis. The populations from the highest elevations exhibited the highest mean germination rates.
\end{abstract}

Key words: alpine species, endemic, calcareous cliffs vegetation, protected flora.

\section{Introduction}

Campanula raineri Perp. is a stenoendemic chasmophyte of the Italian calcareous Prealps, typical of the Physoplexido comosae - Potentillenion caulescentis plant association (Aeschimann \& al. 2004; Pignatti \& al. 2018). It grows with scattered populations in a limited area among Lake Como, Lake Garda and the valley of Valtellina (Lombardy Region and Trentino Alto Adige/Südtirol, northern Italy), occupying crevices on cliffs and screes between 1000 and $2000 \mathrm{~m}$ a.s.l. (Pignatti \& al. 2018; S.Villa personal observations). The species is legally protected by regional law (annex $\mathrm{C}$ of Deliberazione della Giunta Regionale. 27.01.2010 - n. 8/11102) by inclusion in the list of species in need of strict protection, along with 10 congeneric species. For the purposes of this study, a collection permit was granted by Regione Lombardia (Decreto n. 9336).

This perennial species reproduces both by rhizomes creeping between rock crevices and by seed. Between July and August, rosettes produce dense clusters of flowers that develop into trilocular, poricidal capsules (Pignatti \& al. 2018; Fig. 1A). Between September and October, mature seeds are released.

Ex-situ cultivation of $C$. raineri has already been carried out by the University of Insubria (B.E.L. Cerabolini \& R.M. Ceriani, unpublished data) with the aim of reintroducing the species to Monte Barro regional park (LC, Italy), where it seems to have become 
locally extinct (Brusa 2005). Reintroduction was successful, but more than 15 years later the re-established plants are no longer evident (S.Villa, personal observations).

This study investigated the germination response of seeds from nine different populations of C. raineri scattered across the geographical range of the species. Ripe capsules were collected during dry weather, transported in paper bags and stored in a drying room (relative humidity: $15-17 \%$ ) at the Lombardy Seed Bank (LSB at the Centro Flora Autoctona, Galbiate -LC-, CFA) for 4 months before performing in vitro sowing. Dry seeds were cleaned by hand and with an Aspirator \& Gravity Separator machine (PETKUS Selecta Zig-Zag aspirator, v ZIG7-EN-11).

\section{Campanula raineri Perp. (Campanulaceae)}

\section{Accession data}

It: $\quad$ Moggio (LC), Piani di Artavaggio (WGS84: $45.94221^{\circ} \mathrm{N}, 9.53642^{\circ} \mathrm{E}$ ), calcareous screes, 1789 m a.s.1, 30 Sep 2020, S. Villa, (LC930, Lombardy Seed Bank)

It: Taleggio (BG), Monte Venturosa (WGS84: $45.92875^{\circ} \mathrm{N}, 9.61602^{\circ} \mathrm{E}$ ), calcareous cliffs, 1885 m a.s.1, 15 Sep 2020, S. Villa, (BG970, Lombardy Seed Bank)

It: Canzo (CO), Sasso Malascarpa (WGS84: $45.85045^{\circ} \mathrm{N}, 9.31893^{\circ} \mathrm{E}$ ), calcareous cliffs, 1159 m a.s.1, 9 Sep 2020, S. Villa, (CO745, Lombardy Seed Bank)

It: $\quad$ Lecco (LC), Resegone (WGS84: $45.86306^{\circ} \mathrm{N}, 9.46189^{\circ} \mathrm{E}$ ), calcareous cliffs, 1645 m a.s.1, 14 Sep 2020, S. Villa, (LC931, Lombardy Seed Bank)

It: $\quad$ Mandello del Lario (LC), Grigna Meridionale (WGS84: $45.91710^{\circ} \mathrm{N}, 9.39356^{\circ} \mathrm{E}$ ), calcareous cliffs, 1728 m a.s.1, 18 Sep 2020, S. Villa, (LC932, Lombardy Seed Bank)

It: $\quad$ Valleve (BG), Monte Cavallo (WGS84: $46.03302^{\circ} \mathrm{N}, 9.69508^{\circ} \mathrm{E}$ ), calcareous cliffs, 2130 m a.s.1, 15 Sep 2020, S. Villa, (BG971, Lombardy Seed Bank)

It: $\quad$ Colere (BG), Pizzo della Presolana (WGS84: $\left.45.95129^{\circ} \mathrm{N}, 10.06785^{\circ} \mathrm{E}\right)$, calcareous cliffs/screes, 1856 m a.s.1, 28 Sep 2020, S. Villa, (BG972, Lombardy Seed Bank)

It: $\quad$ Oltre il Colle (BG), Pizzo Arera (WGS84: $45.92852^{\circ} \mathrm{N}, 9.80295^{\circ} \mathrm{E}$ ), calcareous cliffs/screes, 1934 m a.s.1, 28 Sep 2020, S. Villa, (BG973, Lombardy Seed Bank)

It: $\quad$ Canzo/Valbrona (CO), Corni di Canzo (WGS84: $45.86281^{\circ} \mathrm{N}, 9.32315^{\circ} \mathrm{E}$ ), calcareous cliffs/screes, 1226 m a.s.1, 9 Sep 2020, S. Villa, (CO746, Lombardy Seed Bank)

\section{Germination data}

Pre-treatments: Seed sterilization in $10 \%$ sodium hypochlorite $(\mathrm{NaOCl})$ solution containing a few drops of detergent acting as a surfactant for 5 minutes, followed by six rinses in sterilized distilled water.

Germination medium: sterile agar medium $\left(6 \mathrm{~g} \mathrm{~L}^{-1}\right)$ enriched with sucrose $\left(20 \mathrm{~g} \mathrm{~L}^{-1}\right)$ for carbohydrate supply, Murashige \& Skoog (1962) mineral salts $\left(2.2 \mathrm{~g} \mathrm{~L}^{-1}\right)$ for nutrient supply and activated charcoal powder $\left(0.5 \mathrm{~g} \mathrm{~L}^{-1}\right)$ to prevent the accumulation of toxic secondary metabolites. The $\mathrm{pH}$ was adjusted to 5.7 and the medium was autoclaved at $121^{\circ} \mathrm{C}$ and $1 \mathrm{~atm}$ for 20 minutes. Filter-sterilised gibberellic acid $\left(\mathrm{GA}_{3} ; 40 \mathrm{mg} \mathrm{L}^{-1}\right)$ was added to the substrate when a non-contact infra-red thermometer determined that the substrate had cooled to $60^{\circ} \mathrm{C}$ (modified from Cerabolini \& al. 2004). 
Sample size: 250 seeds for each test $(25 \times 10$ replicates $)$.

\begin{tabular}{cccccccc}
\hline Germination & Thermoperiod & $\begin{array}{c}\text { Photoperiod } \\
\text { [light/dark] }\end{array}$ & $\mathbf{T}_{\mathbf{1}}[\mathbf{d}]$ & $\mathbf{T}_{\mathbf{5 0}}[\mathbf{d}]$ & $\mathbf{T}_{\max }[\mathbf{d}]$ & MTG [d] & Accession \\
\hline $\mathbf{9 0 . 4 \%}$ & alternating $20 / 10^{\circ} \mathrm{C}$ & $16 / 8 \mathrm{~h}$ & 13.2 & 14.5 & 28.0 & 21.2 & BG971 \\
\hline $\mathbf{8 6 . 2 \%}$ & alternating $20 / 10^{\circ} \mathrm{C}$ & $16 / 8 \mathrm{~h}$ & 14.8 & 16.0 & 28.0 & 21.8 & CO746 \\
\hline $\mathbf{8 6 . 1 \%}$ & alternating $20 / 10^{\circ} \mathrm{C}$ & $16 / 8 \mathrm{~h}$ & 10.3 & 14.3 & 28.0 & 21.1 & BG973 \\
\hline $\mathbf{8 3 . 2 \%}$ & alternating $20 / 10^{\circ} \mathrm{C}$ & $16 / 8 \mathrm{~h}$ & 14.0 & 15.1 & 28.0 & 21.5 & LC932 \\
\hline $\mathbf{8 2 . 7 \%}$ & alternating $20 / 10^{\circ} \mathrm{C}$ & $16 / 8 \mathrm{~h}$ & 15.6 & 16.7 & 28.0 & 22.4 & LC930 \\
\hline $\mathbf{8 2 . 6 \%}$ & alternating $20 / 10^{\circ} \mathrm{C}$ & $16 / 8 \mathrm{~h}$ & 13.5 & 15.2 & 28.0 & 21.6 & LC931 \\
\hline $\mathbf{8 2 . 3 \%}$ & alternating $20 / 10^{\circ} \mathrm{C}$ & $16 / 8 \mathrm{~h}$ & 14.0 & 15.1 & 28.0 & 21.5 & BG972 \\
\hline $\mathbf{8 0 . 2} \%$ & alternating $20 / 10^{\circ} \mathrm{C}$ & $16 / 8 \mathrm{~h}$ & 15.0 & 16.2 & 28.0 & 22.0 & BG970 \\
\hline
\end{tabular}

\section{Observations}

The low concentration $(0.6 \%)$ of agar in the germination medium was chosen because lower agar gel concentrations exhibit lower surface tensions (Ichinose \& Ura 2020), and thus greater contact between substrate and seed, which should facilitate resource acquisition by the seed. Moreover, in vitro germination of other Campanula species has previously been achieved using either half-strength or full-strength Murashige \& Skoog (1962) medium (MS) and relatively low concentrations $(<1 \%$ ) of agar (Airò \& al. 2009; Seglie \& al. 2012; Stamenkovic \& al. 2012; Frattaroli \& al. 2013). Here, we supplied nutrients (rather than using water agar) because our aim was to encourage growth after germination, for the production of plants. We used halfstrength MS medium because previous studies suggest that plants dwelling calcareous rock crevices prefer lower concentrations of mineral salts (Prevalek-Kozlina \& al. 1997, 1999). Petri dishes containing sown seeds were incubated in a growth chamber for 28 days and positions randomized once a week. Germination was periodically monitored through weekly counting (Fig. 1B), and samples exhibiting mould development were progressively discarded. Only 17 out of 90 Petri dishes $(<20 \%)$ were discarded due to contamination, and at least 5 replicates per population were preserved. High germination responses $(>80 \%)$ were obtained for all populations under analysis, with the sole exception of Sasso Malascarpa, the population at the lowest altitude, which exhibited a significantly lower mean germination percentage $(69.1 \pm 7.3 \%)$ with respect to all other populations (ANOVA: F-ratio $=4.35, \mathrm{df}=8, p=0.0003$; data not shown). In contrast, the highest germination rates $(>90 \%)$ were evident for seeds from Monte

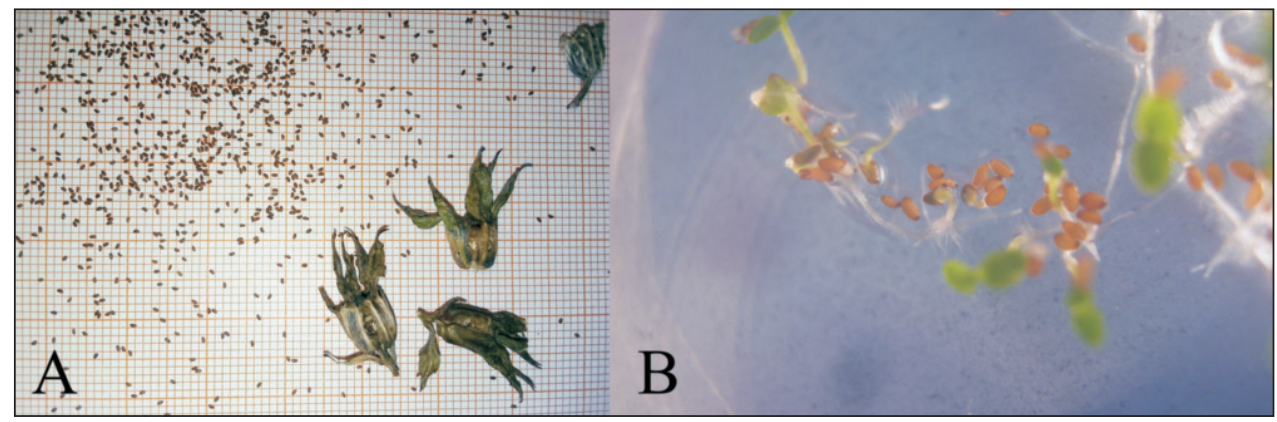

Fig. 1. Mature capsules and seeds (A) and several seedlings (B) of Campanula raineri observed two weeks after sowing on agar medium (photo by S. Villa). 
Cavallo, the population at the highest elevation. A month after the end of the experiment, seedlings were transferred to soil and grown in a greenhouse, while the remaining seeds were deposited in a drying room at the Lombardy Seed Bank (LSB).

\section{References}

Aeschimann, D., Lauber, K., Moser, D. M. \& Theurillat, J.-P. 2004: Flora alpina, 2. - Bologna.

Airò, M., Savona, M., Mascarello, C., De Benedetti, L., Nicoletti, F., Casazza, G., Ruffoni, B. \& Minuto, L. 2009: Analysis of in vitro performances and genetic variability of several Campanula sabatia populations in Italy. - Acta Hortic. 890: 109-112 https://doi.org/10.17660/ActaHortic.2010.860.15

Brusa, G. 2005: Reintroduzione di Campanula raineri Perpenti sul Monte Barro (Lecco). Pp. 79-82 in: Rinaldi, G. \& Rossi, G. (eds), Orti botanici, conservazione e reintroduzione della flora spontanea in Lombardia. Quaderni della Biodiversità, 2. - Bergamo.

Cerabolini, B., De Andreis, R., Ceriani, R. M., Pierce, S. \& Raimondi, B. 2004: Seed germination and conservation of endangered species from the Italian Alps: Physoplexis comosa and Primula glaucescens. - Biol. Conserv. 117: 351-356. https://doi.org/10.1016/j.biocon.2003.12.011

Frattaroli, A. R., Di Martino, L., Di Cecco, V., Catoni, R., Varone, L., Di Santo, M. \& Gratani, L. 2013: Seed germination capability of four endemic species in the Central Apennines (Italy): relationships with seed size. - Lazaroa 34: 43-53.

Ichinose, N. \& Ura, H. 2020: Concentration dependence of the sol-gel phase behavior of agarosewater system observed by the optical bubble pressure tensiometry. - Sci. Rep. 10: 2620. https://doi.org/10.1038/s41598-020-58905-8

Murashige, T. \& Skoog, F. 1962: A revised medium for rapid growth and bioassays with tobacco tissue cultures. - Physiol. P1. 15: 473-497. https://doi.org/10.1111/j.1399-3054.1962.tb08052.x

Pevalek-Kozlina, B., Pavlica, M. \& Vujevic, M. 1999: Micropropagation of Degenia velebitica (Deg.) Hay., a Croatian endemic plant species. - Phyton 39: 293-296.

Pignatti, S., Guarino, R. \& La Rosa, M. (eds) 2018: Flora d'Italia $2^{\circ}$ ed \& Flora digitale, 3. - Milano.

Prevalek-Kozlina, B., Kostovic-Vranjes, V. \& Slade, D. 1997: In vitro propagation of Fibigia triquetra (DC.) Boiss., a rare stenoendemic species. - Pl. Cell Tissue Org. 51: 141-143. https://doi.org/10.1023/A:1005965114749

Seglie, L., Scariot, V., Larcher, F., Devecchi, M. \& Chiavazza, P. M. 2012: In vitro seed germination and seedling propagation in Campanula spp. - Pl. Biosyst. 146(1): 15-23. http://dx.doi.org/10.1080/11263504.2011.578088

Stamenkovic, V., Jelencic, B. \& Tkalec, M. 2012: Propagation and antioxidative enzymes of Campanula velebitica Borbás grown in vitro. - Period. Biol. 114(1): 77-82.

Addresses of the authors:

Sara Villa $^{1 *}$, Roberta M. Ceriani ${ }^{2}$, Bruno E. L. Cerabolini ${ }^{3}$, Simon Pierce ${ }^{1}$,

${ }^{1}$ Dipartimento di Scienze Agrarie e Ambientali - Produzione, Territorio, Agroenergia

(DiSAA), Università degli Studi di Milano, Via G. Celoria 2, 20133, Milano, Italy.

E-mail: sara.villa1@unimi.it; simon.pierce@unimi.it

${ }^{2}$ Centro Flora Autoctona (Native Flora Centre of Lombardy); CFA, c/o Parco Monte Barro, via Bertarelli 11, I-23851, Galbiate, LC, Italy. E-mail: centroflora@parcobarro.it ${ }^{3}$ Dipartimento di Biotecnologie e Scienze della Vita (DBSV), Università dell'Insubria, via J.H. Dunant 3, 21100 Varese, Italy. E-mail: bruno.cerabolini@uninsubria.it

* Corresponding author 